\title{
Friedel phases and phases of transmission amplitudes in quantum scattering systems
}

\author{
Tooru Taniguchi and Markus Büttiker \\ Département de Physique Théorique, Université de Genève, CH-1211, Genève 4, Switzerland
}

(March 1, 2018)

\begin{abstract}
We illustrate the relation between the scattering phase appearing in the Friedel sum rule and the phase of the transmission amplitude for quantum scatterers connected to two one-dimensional leads. Transmission zero points cause abrupt phase changes $\pm \pi$ of the phase of the transmission amplitude. In contrast the Friedel phase is a continuous function of energy. We investigate these scattering phases for simple scattering problems and illustrate the behavior of these models by following the path of the transmission amplitude in the complex plane as a function of energy. We verify the Friedel sum rule for these models by direct calculation of the scattering phases and by direct calculation of the density of states.
\end{abstract}

\section{INTRODUCTION}

The phase is an essential concept in quantum scattering theory. Some key fesults and techniques, such as partial wave expansion, 1 and the Friedel sum rule日 depend in an explicit manner on the scattering phase. The Friedel sum rule connects the density of states to the charge (or a charge difference) of the syster yia the phase of the eigenvalues of the scattering matrix. E $^{5}$ Since the Friedel phase is related to the density of states it is also connected to thermodynamic statistical mechanics quantities 6 like the persistent current In addition to the total density of states the scattering phases als play an important role in the partial density of states 8 (density of states with a preselection or postselection of the incident or exiting quantum channel) and in transport coefficients like capacitances 9 and charge relaxation resistances. 10

The principal aim of this work is to investigate the behavior of the phase in simple scattering problems as they frequently occur in mesoscopic physics. In particular we would like to understand which phases are continuous functions of external parameters (Fermi energy, magnetic field or Aharonov-Bohm flux) and which phases are permitted to exhibit jumps as a function of the external parameters. We consider coherent quantum scattering systems connected to two semi-infinite, one-channel leads in the absence of a magnetic field and without spinorbit scattering. Consider for instance the transmission amplitude $t$ which determines the transmitted current amplitude if there is an incident current of unit amplitude. The transmission amplitude can be expressed in term of its modulus $|t|$ and its phase $\theta^{(t)}$,

$$
t \equiv|t| e^{i \theta^{(t)}} .
$$

If as a function of energy $|t|$ is always positive the path of the transmission amplitude will encircle the origin of the complex plane but always stay at a finite distance from it. An example of such a behavior is shown in Fig. 1. As

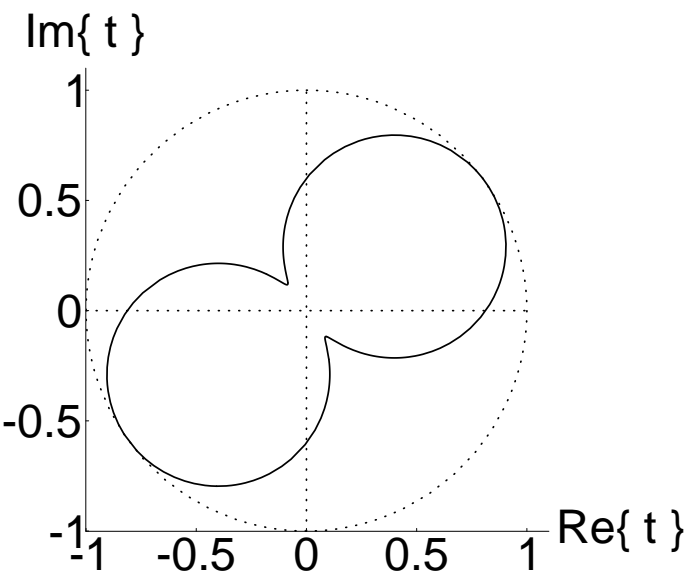

FIG. 1. Transmission amplitude as a function of energy for a resonant double-barrier.

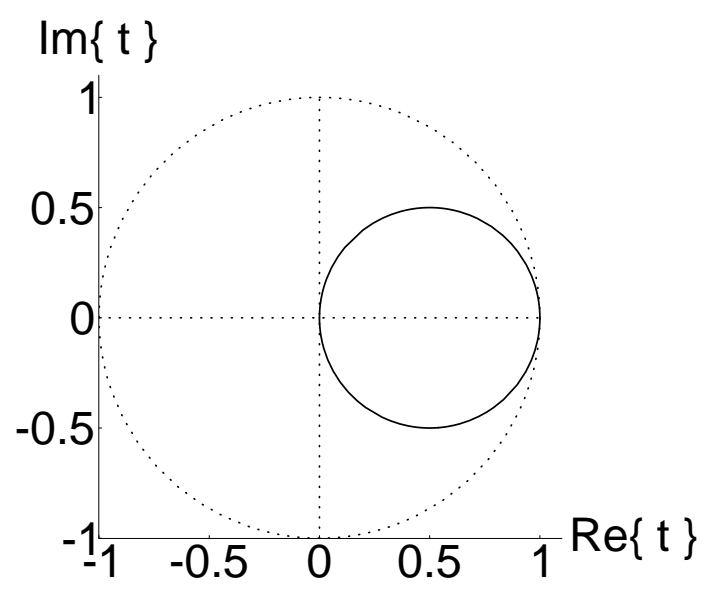

FIG. 2. Transmission amplitude as a function of energy for a wire with a side branch. 
a consequence the phase $\theta^{(t)}$ is a continuous function of the energy. If on the other hand $|t|$ is zero for a certain value of the external parameter, the path of the transmission amplitude in the complex plain will necessarily pass through the origin. As a consequence the phase $\theta^{(t)}$ will jump at this energy by $\pi$. Such a behavior is shown in Fig. 2. A specific model giving rise to the behavior shown in Fig. 11 is the transmission amplitude of a simple one-dimensional resonant tunnel barrier structure. The transmission probability never vanishes and the phase $\theta^{(t)}$ is a continuous function of energy which increases in each complete revolution by $2 \pi$. In contrast, Fig. 2 shows the transmission amplitude for a simple model of a side branch of finite length attached to a perfect wire. The transmission amplitude as function of energy passes through zero and the phase which increases by $\pi$ through each revolution also exhibits a jump of $\pi$ (or $-\pi)$ bringing the phase back to its origin.

The two models with the very distinctive behavior can be combined and the evolution of the transmission amplitude of such a combined model is shown in Fig. 3. Now the graph shows very many revolutions through the origin and occasionally a revolution around the origin. We believe that this reflects the generic behavior of the transmission amplitude in the complex plane.

Obviously the phase of the transmission amplitude in the second example, Fig. 2, since it is not a continuous function, can not play the role of the scattering phase which is used in the Friedel sum rule. For a system with a density of states $\rho$ there must exist a phase which we denote by $\theta^{(f)}$ and which we call the Friedel phase, such that its energy derivative is directly related to the density of states

$$
\frac{\partial \theta^{(f)}}{\partial E}=\pi \rho
$$

Since the density of states should be a continuous function of the energy for the scattering problems we have in mind, the Friedel phase must also be a continuous function of energy. One aim of our work is to investigate and illustrate the behavior of the different phases $\theta^{(t)}$ and $\theta^{(f)}$ and to investigate their connection to the scattering matrix of the problem.

The problem investigated here is of inferest in connection with experiments by Yacoby et al.11 and Schuster et al.12 In the experiment of Yacoby et al. an AharonovBohm ring with a quantum dot was investigated in a two terminal geometry. In a two terminalgeometry the Aharonov-Bohm effect exhibits a parity: 13 As function of the Aharonov-Bohm flux the conductance is either a local minimum at zero flux (positive parity) or a local maximum (negative parity). In the experiment of Yacoby et al. it was observed that over a sequence of more than a dozen Coulomb blockade peaks the parity changes at each peak in an identical manner. It is observed that the parity is positive to the left of the Coulomb blockade peak and negative to the right of the Coulomb blockade peak. Such a behavior is incompatible with a simple resonant tunneling model Fig. 11 but would be in accordance with the evolution of the transmission amplitude shown in Fig. 2. The second important experimental fact is that the phase drops by $\pi$ between Coulomb blockade peaks as shown in the experiment of Schuster et al. Again this behavior is compatible with Fig. 2 but not with Fig. 1. A number of efforts 1328 have been made to explain the fact that the behavior of the parity is the same at each peak. Ref. 13 suggested a screening effect, Ref. 15 alluded to degeneracies, Refs. 17,20,21 and 24 proposed asymmetric deformation of the dot and repeated tunneling through exactly the same state. The same mechanism due to a dot which-is only semi-chaotic is supported by Silvestrov and Imry 28 who point to the large variation in the lifetime of states in a semi-chaotic dot. A possible role of zero's of the transmission probability, Fig. 2, was suogoested to us in informal communication by Levy Yeyati29 since found interest in a number of works. 18, 22, 23, 25 27 We mention here especially the work by Ryu and Cho 23 who investigate an AB ring with a dot which is also connected to a side branch. Apart from Ref. 13 the Friedel sum rule has thus far only found interest in the recent work of Lee.27 The work presented here is in very close connection to the papers by Ryu and Cho and the paper by Lee.

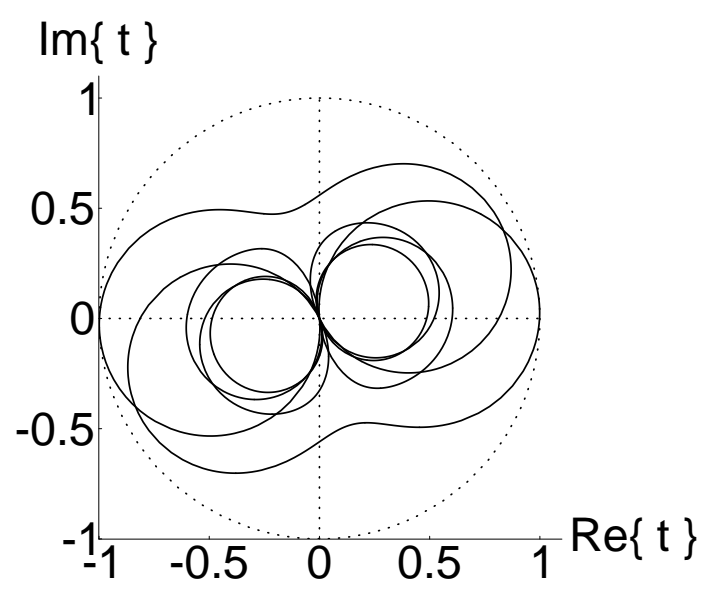

FIG. 3. Transmission amplitude as a function of the particle energy for a model which combines the resonant tunnel barrier and the side branch.

It is possible that the experimental observation of identical behavior over many subsequent conductance peaks is in fact generic (i.e. is observed for a fully chaotic quantum dot) and does require a system with special properties which generates tunneling through the same state. The parity of the Ahronov-Bohm effect was observed in a multi-channel GaAs ring already almost a decade-ago in an experiment by Ford, Washburn and Fowler 30 In 
this experiment the ring is coupled to a back gate and the experiments shows wide regions in the gate voltage magnetic field plane in which the parity of the AharonovBohm effect remains the same.

In this work we investigate the various phases of the scattering matrix and their relation to the phases of the transmission amplitude and the Friedel phase $\theta^{(t)}$ comparing in detail two simple model systems, a onedimensional resonant tunneling problem and a perfect wire with a side branch and briefly discuss also the combination of these two models.

\section{FRIEDEL PHASE AND TRANSMISSION AMPLITUDE PHASE}

We consider systems connected to two semi-infinite, one-dimensional leads. The system itself can be a multidimensional, i. e. a quantum dot. We consider phasecoherent scattering and neglect particle-particle interactions and inelastic scattering effects. In such systems the scattering matrix $S$ in the particle energy $E$ is represented as a $2 \times 2$ matrix in which $S_{j j}$ is the reflection amplitude back into the $j$-th lead for carriers incident in the $j$-th lead $(j=1,2)$ and $S_{j k}$ is the transmission amplitude from the $k$-th lead to the $j$-th lead $(j \neq k, j=1,2, k=1,2)$. The scattering matrix is an unitary matrix, $S^{-1}=S^{\dagger}$. This condition guarantees a conservation of the particle current. Furthermore it implies that the eigenvalues of the scattering matrix $S$ are on the unit circle. Therefore the eigen value of the scattering matrix $S$ can be represented as $e^{2 i \xi_{j}}$ with a real quantity $\xi_{j} \quad(j=1,2)$. Below we show that we obtain the Friedel sum rule if we define the Friedel phase $\theta^{(f)}$ by

$$
\theta^{(f)} \equiv \sum_{j=1}^{2} \xi_{j}
$$

As for any definition of a phase, Eq. (3), defines the Friedel phase only up to a multiple of $\pi$. A unitary $2 \times 2$ matrix can be parameterized as follows,

$$
S=\left(\begin{array}{cc}
i e^{i\left(\theta+\varphi_{1}\right)} \sin \phi & e^{i\left(\theta+\varphi_{2}\right)} \cos \phi \\
e^{i\left(\theta-\varphi_{2}\right)} \cos \phi & i e^{i\left(\theta-\varphi_{1}\right)} \sin \phi
\end{array}\right)
$$

with real phases $\theta, \varphi_{1}, \varphi_{2}$ and $\phi$. The eigenvalues of this matrix $\lambda_{j}=\exp \left(2 i \xi_{j}\right)$ are determined by

$$
\sin \left(2 \xi_{j}-\theta\right)=\sin \varphi_{1} \cdot \cos \phi
$$

The eigenvalues are independent of $\varphi_{2}$. Furthermore if $\xi_{-}$is a solution of Eq. (5) then a second solution is $2 \xi_{+}-\theta=\pi-\left(2 \xi_{-}-\theta\right)$. Consequently the sum of the two phases of these eigenvalues is $2 \xi_{+}+2 \xi_{-}=\pi+2 \theta$. Thus according to Eq. (3) the Friedel phase is given by

$$
\theta^{(f)}=\theta+\frac{\pi}{2}
$$

Apart form a constant $\pi / 2$ the Friedel phase is determined only by the phase $\theta$ of the scattering matrix. In particular, as also emphasized by Lee,27 it would be incorrect to identify the Friedel phase with the argument of the transmission amplitude.

The derivative of the Friedel phase $\theta^{(f)}$ with respect to the particle energy $E$ can be related to the energy derivatives of the scattering matrix. The density of states can also be expressed in terms of the scattering matrix. 5 This gives us a relation which connects the energy derivatives of the Friedel phase and the scattering matrix with the density of states,

$$
\begin{aligned}
\frac{\partial \theta^{(f)}}{\partial E} & =\frac{1}{4 i} \sum_{j=1}^{2} \sum_{k=1}^{2}\left(S_{j k}^{*} \frac{\partial S_{j k}}{\partial E}-\frac{\partial S_{j k}^{*}}{\partial E} S_{j k}\right) \\
& =\pi \rho
\end{aligned}
$$

Integration of this relation over the energy interval $\left[E_{1}, E_{2}\right]$ gives the Friedel sum rule, which thus states that the difference in phase $\theta^{(f)}\left(E_{2}\right)-\theta^{(f)}\left(E_{1}\right)$ is equal to the number of particles $N\left(E_{2}, E_{1}\right)$ multiplied by $\pi$ in the system in this energy interval,

$$
\theta^{(f)}\left(E_{2}\right)-\theta^{(f)}\left(E_{1}\right)=\pi N\left(E_{2}, E_{1}\right)
$$

The Friedel phase is thus a a continuous function of energy (either $E_{2}$ or $E_{1}$ ) since the density of states is a continuous function of energy.

Another phase which is frequently discussed is the argument $\theta^{(t)}$ of the transmission amplitude defined by Eq. (11). Below we will consider only systems with time reversal invariance. For such systems the scattering matrix is symmetric $S_{12}=S_{21}$ and consequently $\varphi_{2}=0$ or $\varphi_{2}=\pi$. To be definite, we take, $\varphi_{2}=0$. In this case the phase of the transmission amplitude is given by

$$
\theta^{(t)}=\theta+\pi \Theta(\cos \phi)
$$

where $\Theta(x)$ is the step function of $x$. In contrast to the Friedel phase, the phase of the transmission amplitude is thus in general not a continuous function of energy, but exhibits a jump of $\pi$ when $\cos \phi$ changes sign. The Friedel phase and the transmission amplitude phase are not completely independent. From Eqs. (6) and (9) we find

$$
\theta^{(t)}=\theta^{(f)}-\frac{\pi}{2}+\pi \Theta(\cos \phi)
$$

Differentiating Eq. (10) with respect to energy (or any other variable of interest) we find,

$$
\Delta \theta^{(t)}=\Delta \theta^{(f)} \pm \pi \delta(\cos \phi) \Delta \cos \phi
$$

where we have used the abbreviation $\Delta=d / d E$. Eq. (11) shows that only if the condition $\cos \phi \neq 0$ is satisfied for all values of the parameters is the phase of the transmission amplitudes equal to the Friedel phase. The 
condition $\cos \phi \neq 0$ means that the transmission probability $|t|^{2}$ is nowhere equal to zero. Therefore it is the existence of zero points of the transmission probability which is at the origin of the difference of the Friedel phase and the phase of the transmission amplitude.

To be more precise, in addition to a zero point in the transmission probability, it is also required that the energy derivative of $\partial \cos \phi / \partial E$ is non-zero at the transmission zero-points. To see this, we consider phase changes due to the energy $E$ only, and assume that the number of zero points of the transmission probability is finite or countable. The zero points of the transmission probability determines a sequence of energies which we denote by $E^{(n)}, n=1,2, \ldots$ Using Eqs. (7) and (11) we obtain

$$
\frac{\partial \theta^{(t)}}{\partial E}=\pi \rho+\pi \sum_{n} \operatorname{sgn}\left(\left.\frac{\partial \cos \phi}{\partial E}\right|_{E=E^{(n)}}\right) \delta\left(E-E^{(n)}\right) .
$$

where the function $\operatorname{sgn}(x)$ of $x$ is the $\operatorname{sign}$ function. It may be noted that the second term of the right-hand side of Eq. (12) is zero even at a transmission zero point if $\partial \cos \phi / \partial E$ is zero at such a point. However, we expect that such cases are unlikely, and we proceed by assuming that $\partial \cos \phi / \partial E$ is not zero at any transmission zero point.

To summarize: The Friedel phase is a continuous function of energy. On the other hand, the argument of the transmission amplitude, might exhibit jumps. These conclusions agree with Lee.27

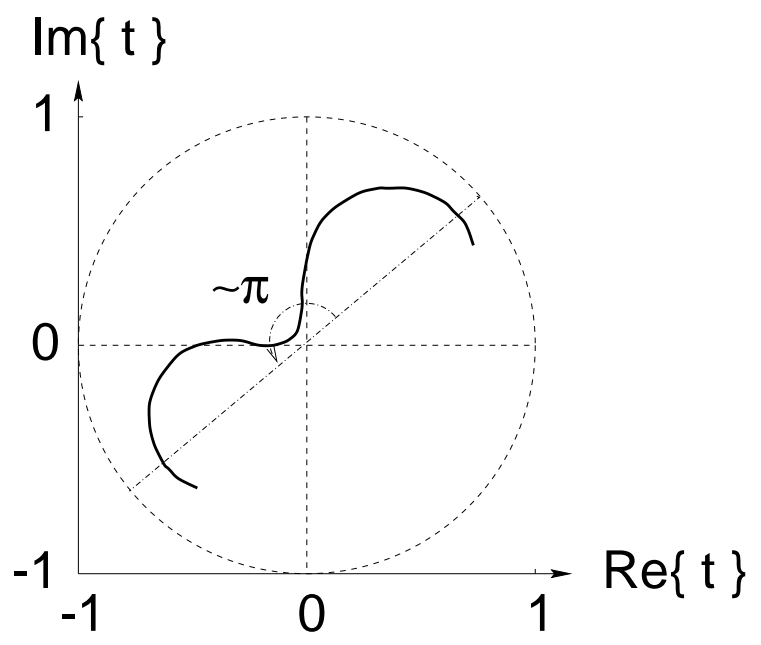

FIG. 4. Schematic representation of the path of the transmission amplitude as a function of the particle energy for the case that there is no transmission zero. The energy interval covered is that needed for the transition from one resonant peak (in the transmission probability) to the next resonant peak. The phase changes by about $\pi$.

\section{EVOLUTION OF THE PATH OF THE TRANSMISSION AMPLITUDE CONNECTING CONSECUTIVE RESONANCES}

We now discuss, from a general point of view, the conditions which lead to the behavior shown in Figs. 1, 2 and 3. We consider a set of parameters for which the transmission probability shows a series of peaks as function of energy. We now ask: What portion of the path of the transmission amplitude shown in Fig. 1 and Fig. 2 is traced out if we increase the energy from its value at a peak in the transmission probability to a value corresponding to the subsequent peak in the transmission probability? Let us denote the energy of the $n$-th peak in the transmission probability by $\mathcal{E}_{n}$. In addition, let us consider the condition

$$
\int_{\mathcal{E}_{n}}^{\mathcal{E}_{n+1}} d E \rho \sim 1
$$

which according to the Friedel sum rule implies that one particle is added to the scattering region. Using this condition and integrating both sides of Eq. (12) with respect to the energy from $\mathcal{E}_{n}$ to $\mathcal{E}_{n+1}$ we find

$$
\theta^{(t)}\left(\mathcal{E}_{n+1}\right)-\theta^{(t)}\left(\mathcal{E}_{n}\right) \sim \begin{cases}\pi & \text { case } \mathcal{A} \\ 0 \text { or } 2 \pi & \text { case } \mathcal{B}\end{cases}
$$

In case $\mathcal{A}$ there is no transmission zero point in the energy interval $\left(\mathcal{E}_{n}, \mathcal{E}_{n+1}\right)$, and in case $\mathcal{B}$ there is a transmission zero point in the energy interval $\left(\mathcal{E}_{n}, \mathcal{E}_{n+1}\right)$. In case $\mathcal{A}$ the phase $\theta^{(t)}$ evolves by $\pi$ through the consecutive resonant peaks, so that the resulting path of the transmission amplitude is as shown in Fig. A. On the other hand, in case $\mathcal{B}$ the phase $\theta^{(t)}$ of the transmission amplitude increases by $2 \pi$ (or 0 ) between the consecutive resonant peaks, so that the trace of the transmission amplitude is as shown in Fig. 5 .

It is clear that Fig. 1 is composed of two paths of the type shown in Fig. 14, and Fig. 2 is the result of a path of the type shown in Fig. 5. Consequently, there is a profound difference in the behavior of these two systems: Whereas, for instance, in a double-barrier scattering problem, we need to increase the energy over two consecutive states to re-arrive at the starting point, for the wire with a side branch an energy increase over one state only is sufficient to bring the system back to the same point. We also remark that Fig. 3 is composed of combinations of the paths shown in Figs. 1 and 5 .

Clearly it would be desirable to classify all the possible paths that are taken in the complex plane as we proceed from one transmission peak to another. Here we have emphasized only two paths, namely those shown in Fig. 4 and 5. These two possibilities are a direct consequence of the condition Eq. (13), but of course there might exist scattering problems which do not obey this condition.

To summarize this Section: We have shown that the addition of a particle to the system can lead to at least 
two very distinct paths of the transmission amplitude in the complex plane. For paths of type $\mathcal{A}$ (see Fig. 4 ) the parity of the Aharonov-Bohm effect in a two terminal geometry is out of phase on consecutive resonant peaks, whereas for a scatterer of type $\mathcal{B}$ (see Fig. 5) the Aharonov-Bohm oscillations are in phase. With respect to the experiment of Yacoby et al. it is an in-phase behavior that is needed to explain the data.

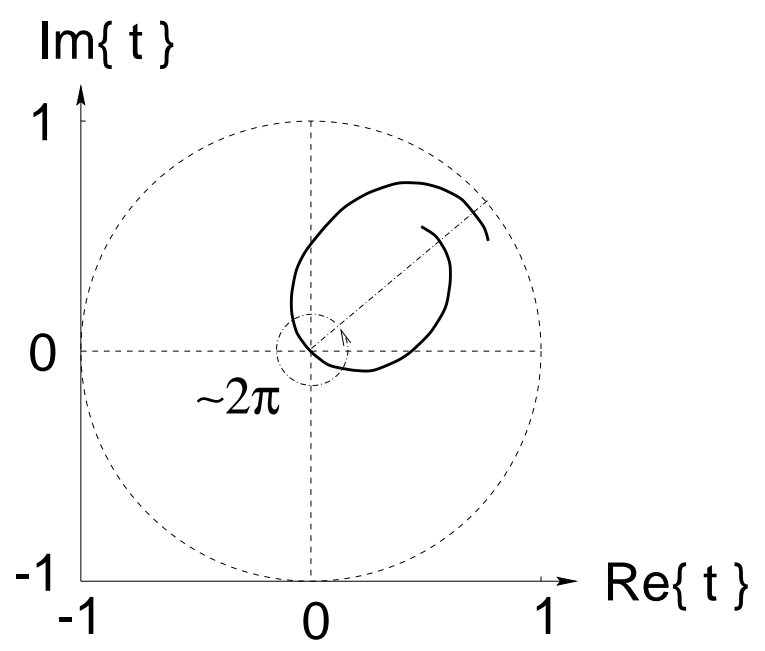

FIG. 5. Schematic representation of the path of the the transmission amplitude as a function of the particle energy for the case that there is a transmission zero. The energy interval covered is that needed for the transition from one resonant peak (in the transmission probability) to the next resonant peak. The phase changes by about $2 \pi$.

\section{EXAMPLES}

In this section we present the calculations which lead to Figs. 1, 2 and 3 for the transmission amplitude. In addition we present results for the density of states as a function of energy which we compare with the transmission probability.

\section{A. The resonant double-barrier}

The first example is a one-dimensional double-barrier model, which consists of two consecutive potential barriers which scatter particles moving along the $x$-axis. A schematic illustration is shown in Fig. 6 .

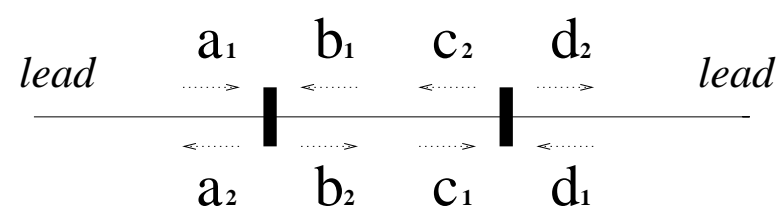

FIG. 6. Current amplitudes in the double-barrier model.

We assume that the two potential barriers in this model are identical and that each potential barrier is symmetric with respect to particles incident from the left and the right. The current amplitudes $a_{j}, b_{j}, c_{j}$ and $d_{j}, j=1,2$ which are shown in Fig. 6, are connected by the following relations,

$$
\begin{aligned}
& \left(\begin{array}{l}
a_{2} \\
b_{2}
\end{array}\right)=\left(\begin{array}{ll}
\tilde{r} & \tilde{t} \\
\tilde{t} & \tilde{r}
\end{array}\right)\left(\begin{array}{l}
a_{1} \\
b_{1}
\end{array}\right) \\
& \left(\begin{array}{l}
c_{2} \\
d_{2}
\end{array}\right)=\left(\begin{array}{ll}
\tilde{r} & \tilde{t} \\
\tilde{t} & \tilde{r}
\end{array}\right)\left(\begin{array}{l}
c_{1} \\
d_{1}
\end{array}\right) .
\end{aligned}
$$

Here, $\tilde{r}$ and $\tilde{t}$ are the reflection and the transmission amplitude of the potential barriers, respectively. The amplitudes in the well are related by $b_{1}=\tau c_{2}$ and $c_{1}=\tau b_{2}$, where $\tau=\exp (i \varphi)$ is the transmission amplitude of the well and $\varphi$ the phase accumulated by a one time traversal of the well.

We have to find the scattering matrix $S$ which relates the outgoing current amplitudes $a_{2}, d_{2}$ to the incoming current amplitudes $a_{1}, d_{1}$. A little algebra gives

$$
S=\left(\begin{array}{cc}
\tilde{r} & 0 \\
0 & \tilde{r}
\end{array}\right)+\frac{\tilde{t}^{2} \tau}{1-\tilde{r}^{2} \tau^{2}}\left(\begin{array}{cc}
\tilde{r} \tau & 1 \\
1 & \tilde{r} \tau
\end{array}\right)
$$

To proceed we parameterize the scattering matrix of the single barrier also in terms of angular variables (see Eq. (ब) . We take $\tilde{r}=i e^{i \tilde{\theta}} \sin \tilde{\phi}$ and $\tilde{t}=e^{i \tilde{\theta}} \cos \tilde{\phi}$ with real angles $\tilde{\theta}$ and $\tilde{\phi}$. In our model the potential is uniform (except for the two barriers) and $\varphi$ is thus connected to the energy $E$ of a scattering particle via $\varphi=k l$ where $l$ is the distance between the potential barriers and $k=\sqrt{2 m E} / \hbar$ is the wave vector of the particle away from the barriers. We assume that the quantities $\tilde{\theta}$ and $\tilde{\phi}$ are independent of the energy $E$. This implies in particular that there is no particle density inside each potential barrier. With these specifications the transmission amplitude $t$ is given by

$$
t=\frac{e^{i(k l+2 \tilde{\theta})} \cos ^{2} \tilde{\phi}}{1+e^{2 i(k l+\tilde{\theta})} \sin ^{2} \tilde{\phi}} .
$$

The transmission amplitude is a function of energy only through the energy dependence of the wave vector $k$. This result is used to give the transmission amplitude in the complex plane as a function of energy in Fig. 1. The parameters chosen for Fig. 11 are $\tilde{\theta}=2.2$ and $\tilde{\phi}=2.1$.

\section{B. Phases and density of states in the double-barrier model}

For comparison with the wire connected to a side branch, we now examine the phases in the double barrier 
scattering problem and the density of states. To find the Friedel phase we use Eq. (7) and find from Eqs. (17),

$$
\frac{\partial \theta^{(f)}}{\partial E}=\frac{\partial \varphi}{\partial E} \frac{1-|\tilde{r}|^{4}}{\left|1-\tilde{r}^{2} \tau^{2}\right|^{2}}
$$

The derivative of the phase $\theta^{(t)}$ of the transmission amplitude is found from $\tan \theta^{(t)}=\operatorname{Im}\{t\} / \operatorname{Re}\{t\}$,

$$
\frac{\partial \theta^{(t)}}{\partial E}=\left\{1+\left(\frac{\operatorname{Im}\{t\}}{\operatorname{Re}\{t\}}\right)^{2}\right\}^{-1} \frac{\partial}{\partial E} \frac{\operatorname{Im}\{t\}}{\operatorname{Re}\{t\}} .
$$

Substituting Eq. (18) into Eq. 20 we can show that for the resonant double barrier the derivatives of the two phases are identical,

$$
\frac{\partial \theta^{(t)}}{\partial E}=\frac{\partial \theta^{(f)}}{\partial E} .
$$

This result just restates Eq. (11) for the double-barrier model. The phase $\theta=\theta^{(t)}=\theta^{(f)}-\pi / 2$ for the double barrier model as a function of $k l$ (wave-vector times well width) is shown in Fig. 7. The specific parameters are $\tilde{\theta}=2.2$ and $\tilde{\phi}=2.1$. Thus the phase shows a step like behavior. It is nearly constant as a function of $k$ and increases sharply when $k$, respectively the energy $E$, coincides with a resonant state. To show this, we now examine the density of states.

For a perfect one-dimensional wire the density of states per unit length and in a small interval of wave vectors is $d n / d k=1 /(2 \pi)$ for carriers moving to the right. Since $d E / d k=\hbar v$ where $v$ is the velocity of the carrier, the density of states per unit length and in a small energy interval is $\nu \equiv d n / d E=1 /(h v)$. We are interested in the density of states in the region between the two barriers. Denoting the scattering states with unit amplitude of incident carriers from the left by $\Phi_{1}(x)$ and the scattering state of carriers with unit amplitude of carriers incident from the right by $\Phi_{2}(x)$, the local density of states can be expressed by 31

$$
\nu(x)=\sum_{\mu=1}^{2} \frac{1}{h v}\left|\Phi_{\mu}(x)\right|^{2} .
$$

The density of states in the well region (between the two barriers) is the integral over the local density of states $\rho=\int_{-l / 2}^{l / 2} d x \nu(x)$. With an explicit calculation of the scattering states, we obtain

$$
\rho=\frac{2 l}{h v}\left|\frac{\tilde{t}}{1-\tilde{r}^{2} \tau^{2}}\right|^{2}\left(1+|\tilde{r}|^{2}+2 \operatorname{Re}\{\tilde{r} \tau\} \frac{\sin \varphi}{\varphi}\right) .
$$

Here, for simplicity, we assume that the phase increment of a barrier traversal is zero. We have considered here the density of states which are obtained in terms of energy derivatives of phases or scattering matrices (as it is widely done). Such derivatives do not naturally lead to an answer for a spatially local density of states. The density of states in the region between the two barriers, is however, a local question. A rigorous procedure to obtain local densities is via derivatives of phases and scattering matrices with respect to local potentials. 1 The discussion given here (in terms of energy derivatives) is correct only up to WKB like corrections. The last term in Eq. 23. contains a factor $1 /(k l)$ and is thus small for wells which are much larger than a wave length. Neglecting this term we obtain,

$$
\bar{\rho}=\frac{2 l}{h v} \frac{1+\sin ^{2} \tilde{\phi}}{\cos ^{2} \tilde{\phi}+4 \tan ^{2} \tilde{\phi} \cdot \cos ^{2}(\tilde{\theta}+k l)} .
$$

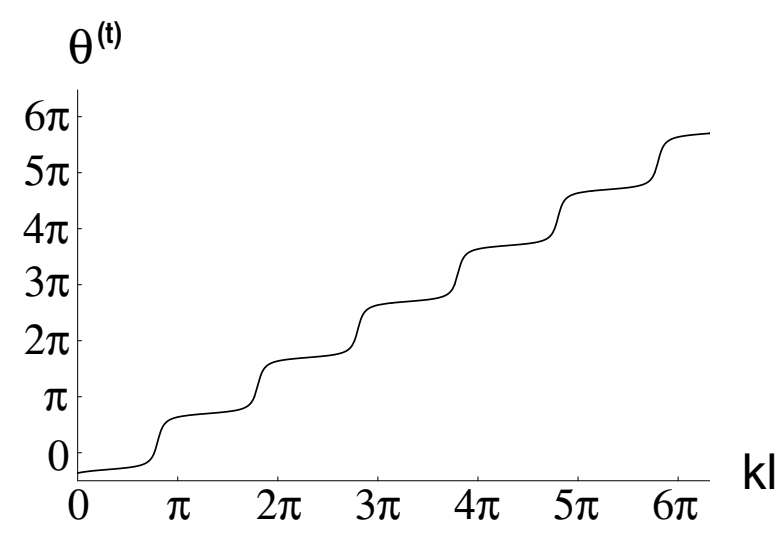

FIG. 7. Phase of the transmission amplitude as a function of $k l(=l \sqrt{2 m E} / \hbar)$ for the double-barrier model.

As is well known, for the case of opaque barriers $(\tilde{\theta} \rightarrow \pi / 2$ and $\tilde{\phi} \rightarrow \pi / 2)$ the density of states becomes a series of delta functions $\lim _{\tilde{r} \rightarrow-1} \bar{\rho}=\sum_{n=1}^{+\infty} \delta\left(E-\pi^{2} \hbar^{2} n^{2} /\left(2 m l^{2}\right)\right)$ which coincide with the peaks of perfect transmission. For wells much wider than the wavelength, we find from Eq. (24) that the peaks in the density of states are at the energies

$$
E_{n}=\frac{\pi^{2} \hbar^{2}}{2 m l^{2}}\left\{n-\frac{1}{\pi}\left(\tilde{\theta}-\frac{\pi}{2}\right)\right\}^{2} .
$$

Here $n$ takes any integer values larger than $(2 \tilde{\theta}-\pi) /(2 \pi)$. Comparison of Eq. (19) with (24) leads to $\partial \theta^{(f)} / \partial E=$ $\pi \bar{\rho}, \mathrm{i}$. e. the Friedel sum rule in the double-barrier model.

Using Eqs. (18) and (24) we obtain

$$
|t|^{2}=\frac{|\tilde{t}|^{2}}{1+|\tilde{r}|^{2}} \frac{\pi \hbar v}{l} \bar{\rho}
$$

a relation between the density of state $\bar{\rho}$ and the transmission probability $|t|^{2}$. This relation implies that the energy values $\mathcal{E}_{n}$ which determine the peaks in the transmission probability coincide with the energies $E_{n}$ of the 
peaks in the density of states. Using this fact and Eq. (24) we can show

$$
\int_{\mathcal{E}_{n}}^{\mathcal{E}_{n+1}} d E \bar{\rho}=1
$$

In particular, Eq. (13) applies to the resonant doublebarriers with well widths large compared to the wave length. Fig. 8 shows the transmission probability and the density of state as a function of $k l$ for the doublebarrier for the parameters $\tilde{\theta}=2.2$ and $\tilde{\phi}=2.1$. We emphasize this behavior of the double barrier, since as we show in the next section, a wire with a side branch, exhibits a transmission probability and a density of states which do not peak at the same energy.

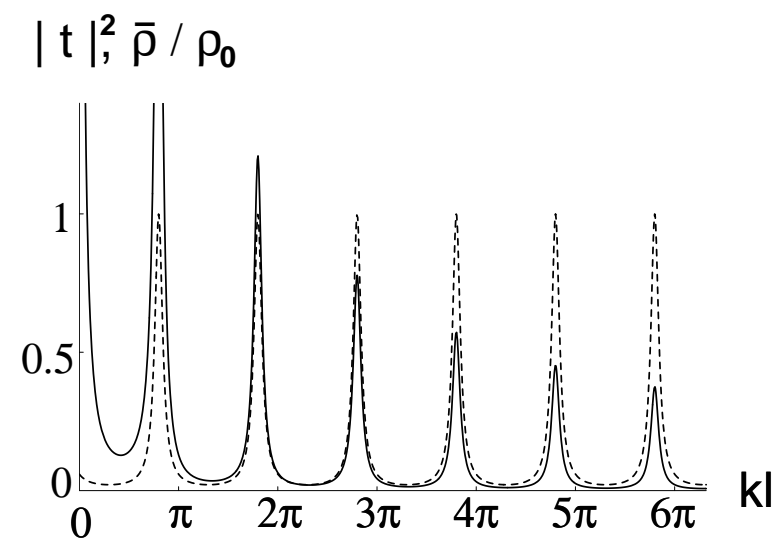

FIG. 8. Transmission probability (the dashed line) and the density of states $\bar{\rho}$ (the solid line) in units of $\rho_{0} \equiv m l^{2} /\left(\pi \hbar^{2}\right)$ as a function of $k l(=l \sqrt{2 m E} / \hbar)$ for the double-barrier.

\section{Wire with a side branch}

The second example which we investigate is a perfect one-dimensional wire with a side branch which we also call the stub model. Such models have already a long history. The conductance of a wire with a side branch was investigated in Refs. 34,35 and 38. Refs. 36 and 37 considered charging effects in structures with side branches. In the context of the present work the phase behavior of the transmission-amplitude has been investigated by Deo and Jayannavar 1839 and Ryu and Cho 23 However, neither of these two works makes the distinction between the Friedel phase and the phase of the transmission amplitude. Below we investigate these two phases for a perfect wire to which a side branch of length $l^{\prime}$ is attached. A schematic illustration of this system is shown in Fig. 9 .

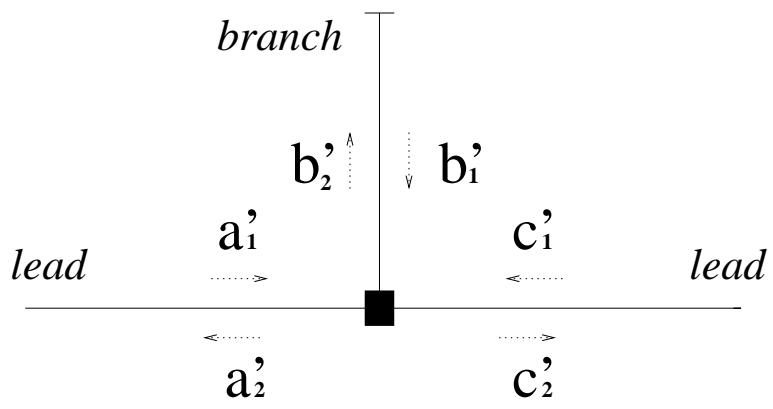

FIG. 9. Current amplitudes for the model of a wire with a side branch.

The junction between the wire and the side branch is described by a wave splitter.32 33 We consider the timereversal invariant case only and consider a splitter which is symmetric with respect to carriers incident from the left and right lead. Furthermore we assume the potential away from the junction is the same in all branches. The current amplitudes $a_{j}^{\prime}, b_{j}^{\prime}$ and $c_{j}^{\prime}, j=1,2$, which are shown in Fig. 9 are connected by the following relations,

$$
\left(\begin{array}{c}
a_{2}^{\prime} \\
b_{2}^{\prime} \\
c_{2}^{\prime}
\end{array}\right)=\left(\begin{array}{ccc}
\tilde{r}^{\prime} & \varepsilon & \tilde{t}^{\prime} \\
\varepsilon & \sigma & \varepsilon \\
\tilde{t}^{\prime} & \varepsilon & \tilde{r}^{\prime}
\end{array}\right)\left(\begin{array}{c}
a_{1}^{\prime} \\
b_{1}^{\prime} \\
c_{1}^{\prime}
\end{array}\right)
$$

and $b_{1}^{\prime}=\tau^{\prime} b_{2}^{\prime} \equiv e^{i \varphi^{\prime}} b_{2}^{\prime}$ with $\varphi^{\prime}=2 k l^{\prime}+\pi$. Here $k$ is the wave vector of a particle with energy $E$. The constant $\pi$ in $\varphi$ guarantees that the wave function has a node at the upper end of the stub. In Eq. (28), $\tilde{r}^{\prime}$ is the reflection amplitude from a lead to itself, $\tilde{t}^{\prime}$ is the transmission amplitude from a lead to another lead, $\sigma$ is the reflection amplitude from the stub to itself, $\varepsilon$ is the transmission amplitude from a lead to the stub or from the stub to a lead, and $\tau^{\prime}$ is the transmission amplitude by which the particle starting from the junction returns to the junction through the stub.

For the scattering matrix, relating the incident amplitudes on the wire $a_{1}^{\prime}, d_{1}^{\prime}$ to the out-going amplitudes $a_{2}^{\prime}, c_{2}^{\prime}$ in the wire (see Fig. 9) we obtain

$$
S=\left(\begin{array}{cc}
\tilde{r}^{\prime} & \tilde{t}^{\prime} \\
\tilde{t}^{\prime} & \tilde{r}^{\prime}
\end{array}\right)+\frac{\varepsilon^{2} \tau^{\prime}}{1-\sigma \tau^{\prime}}\left(\begin{array}{ll}
1 & 1 \\
1 & 1
\end{array}\right)
$$

Here the first matrix on the right hand side arises from direct transmission past the side branch and direct reflection at the wave splitter due to the side branch, whereas the second term of the right-hand side of is the contribution due to carriers which enter the stub and thus a undergo multiple scattering process. We assume that the scattering amplitudes $\tilde{r}^{\prime}, \tilde{t}^{\prime}, \sigma$ and $\varepsilon$ are real numbers, $\varepsilon \neq 0$ and are independent of the energy $E$. These assumptions, and the fact that the scattering matrix Eq. (28) must be unitary, demands 33

$$
\tilde{r}^{\prime}=\left(\lambda_{1}+\lambda_{2} \sqrt{1-2 \varepsilon^{2}}\right) / 2
$$




$$
\begin{aligned}
& \tilde{t}^{\prime}=\left(-\lambda_{1}+\lambda_{2} \sqrt{1-2 \varepsilon^{2}}\right) / 2 \\
& \sigma=-\lambda_{2} \sqrt{1-2 \varepsilon^{2}}
\end{aligned}
$$

where $\lambda_{j}$ is 1 or $-1(j=1,2)$. Depending on the choice of the $\lambda^{\prime} s$ four different wave splitters are obtained. The value of the coupling constant $\varepsilon$ is in the interval $[-1 / \sqrt{2}, 1 / \sqrt{2}]$. Using such a wave splitter leads to a transmission amplitude

$$
t=\frac{-\lambda_{1}+\lambda_{2} \sqrt{1-2 \varepsilon^{2}}}{2} \frac{1+\lambda_{1} e^{2 i k l^{\prime}}}{1-\lambda_{2} \sqrt{1-2 \varepsilon^{2}} e^{2 i k l^{\prime}}} .
$$

The path of this amplitude in the complex plane as a function of energy is shown in Fig. 2 for the case $\lambda_{1}=-1$. The path is a circle through the origin since Eq. (33) implies $\left|t+\lambda_{1} / 2\right|^{2}=1 / 4$. From Eq. (33) it follows that the wire with the stub has zero points of the transmission probability at the energies

$$
E^{(n)}=\frac{\pi^{2} \hbar^{2}}{2 m l^{2}}\left(n-\frac{1+\lambda_{1}}{4}\right)^{2},
$$

$n=1,2, \cdots$. We re-emphasize that in contrast to the case of the double barrier, the origin is included in the path of the transmission amplitude.

\section{Phases and density of states in wire with a side branch}

Let us now investigate the Friedel phase and the phase of the transmission amplitude for the wire with a side branch. Using Eqs. (7) and (29), the derivative of the Friedel phase $\theta^{(f)}$ with respect to the energy $E$ in the wire with a side branch is given by

$$
\frac{\partial \theta^{(f)}}{\partial E}=\frac{\partial \varphi^{\prime}}{\partial E}\left|\frac{\varepsilon}{1-\sigma \tau^{\prime}}\right|^{2} .
$$

As shown in the previous subsection there are zero points in the transmission amplitude as a function of the energy in the stub model, so that abrupt phase changes of the transmission amplitude do occur. Thus we need a limiting procedure to define the phase of the transmission amplitude. To this end we add a small perturbation $\pm \eta, \eta>0$ to the transmission amplitude

$$
\bar{t}_{ \pm} \equiv t \pm \eta
$$

and evaluate the phase of $\bar{t}_{ \pm}$in the limit $\eta \rightarrow+0$. The derivative of the phase $\theta^{(t)}$ of the transmission amplitude with respect to the energy $E$ is thus given by

$$
\frac{\partial \theta^{(t)}}{\partial E}=\lim _{\eta \rightarrow+0}\left\{1+\left(\frac{\operatorname{Im}\left\{\bar{t}_{ \pm}\right\}}{\operatorname{Re}\left\{\bar{t}_{ \pm}\right\}}\right)^{2}\right\}^{-1} \frac{\partial}{\partial E} \frac{\operatorname{Im}\left\{\bar{t}_{ \pm}\right\}}{\operatorname{Re}\left\{\bar{t}_{ \pm}\right\}}
$$

Using our specific result for the transmission amplitude we obtain

$$
\frac{\partial \theta^{(t)}}{\partial E}=\frac{\partial \theta^{(f)}}{\partial E} \pm \lambda_{1} \pi \sum_{n=1}^{+\infty} \delta\left(E-E^{(n)}\right) .
$$

A detailed derivation of Eq. (38) is given in Appendix A. Fig. 10 shows the phase of the transmission amplitude of the wire with a side branch as a function of $k l^{\prime}$. We have chosen the branch of the wave splitter with $\lambda_{1}=-1$, $\lambda_{2}=1$ and a coupling constant $\varepsilon^{2}=0.35$

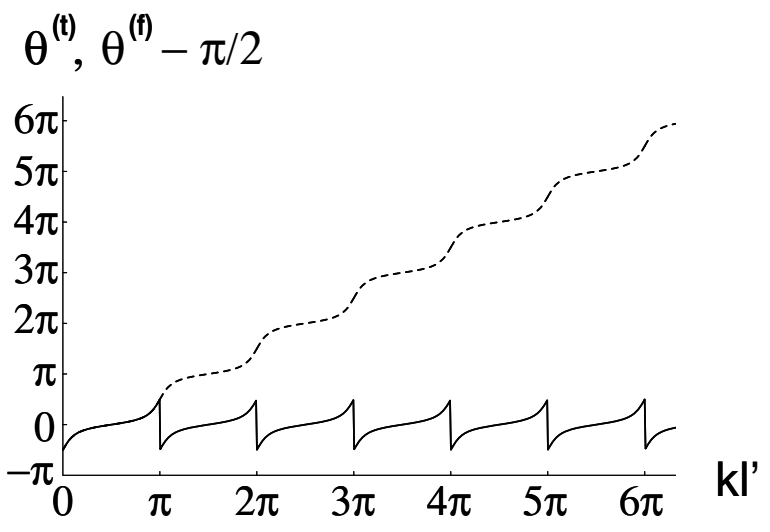

FIG. 10. Phase of the transmission amplitude $\theta^{(t)}$ (solid line) and Friedel phase $\theta^{(f)}-\pi / 2$ (dashed line) as a function of $k l^{\prime}$ for the wire with the side branch.

Let us next investigate the density of states. The wire is taken to be on the $x$-axis, with the splitter located at $x=0$. The stub points along the positive $y$-axis. The splitter is described by an energy independent scattering matrix and can thus be viewed as point like. A scattering state of unit amplitude $\exp \left\{i k_{\mu} x\right\}$ describing particles incident from the $\mu$-th lead $(\mu=1,2)$ gives in the side branch rise to a wave $\Psi_{\mu}(y)$. Here $k_{1}=k$ for a wave incident from the left and $k_{2}=-k$ for a wave incident from the right. The local density of states $\nu(y)$ in the side branch is given by $\nu(y)=\sum_{\mu=1}^{2}\left|\Psi_{\mu}(y)\right|^{2} /(h v)$, and the total density of states is given by the integral of the local density of states over the entire length of the stub, $\rho=\int_{0}^{l^{\prime}} d y \nu(y)$. We find

$$
\rho=\frac{l^{\prime}}{h v}\left|\frac{2 \varepsilon}{1-\sigma e^{i \varphi^{\prime}}}\right|^{2}\left(1+\frac{\sin \varphi^{\prime}}{\varphi^{\prime}-\pi}\right) .
$$

In the WKB limit of interest here, for a side branch much longer than the Fermi wavelength, the second term in the bracket of the right-hand side of Eq. (39) can be neglected. Using Eq. (32) we obtain for a long side branch the density of states

$$
\bar{\rho}^{\prime}=\frac{l^{\prime}}{h v} \frac{4 \varepsilon^{2}}{\left(1-\sqrt{1-2 \varepsilon^{2}}\right)^{2}+4 \sqrt{1-2 \varepsilon^{2}} \cdot \sin ^{2}\left(K_{2} l^{\prime}\right)} .
$$


Here, $K_{j}, j=1,2$ is defined by $K_{j} \equiv k+\left(1-\lambda_{j}\right) \pi /\left(4 l^{\prime}\right)$. For a stub much longer than a wavelength, the energies at which the density of states peaks are given by

$$
E_{n}^{\prime}=\frac{\pi^{2} \hbar^{2}}{2 m l^{\prime 2}}\left(n-\frac{1-\lambda_{2}}{4}\right)^{2}
$$

In the week coupling limit $\varepsilon \rightarrow+0$, using Eq. (40), we obtain $\lim _{\varepsilon \rightarrow+0} \bar{\rho}^{\prime}=\sum_{n=1}^{+\infty} \delta\left(E-E_{n}^{\prime}\right)$. Here the right hand-side is the density of state of a particle confined in the completely isolated stub but taking into account that the phase change at the closed coupler is as dictated by $\lambda_{2}=1$. The comparison of Eq. (35) with Eq. (40) leads to $\partial \theta^{(f)} / \partial E=\pi \bar{\rho}^{\prime}$. We have thus verified the Friedel sum rule for the wire with a side branch.

Let us now show that in this scattering problem (for the splitters with $\lambda_{1} \lambda_{2}=-1$ ) the peaks in the transmission probability do not co-inside with the peaks in the density of states. Using Eq. (33) the transmission probability $|t|^{2}$ can be expressed in the form,

$$
\begin{aligned}
& |t|^{2}= \\
& \frac{\left(1-\lambda_{1} \lambda_{2} \sqrt{1-2 \varepsilon^{2}}\right)^{2} \cos ^{2}\left(K_{1} l^{\prime}\right)}{\left(1-\lambda_{1} \lambda_{2} \sqrt{1-2 \varepsilon^{2}}\right)^{2}+4 \lambda_{1} \lambda_{2} \sqrt{1-2 \varepsilon^{2}} \cdot \sin ^{2}\left(K_{1} l^{\prime}\right)} .
\end{aligned}
$$

Therefore the energy values $\mathcal{E}_{n}, n=1,2, \cdots$ at which the transmission probability peaks are given by

$$
\mathcal{E}_{n}=\frac{\pi^{2} \hbar^{2}}{2 m l^{\prime 2}}\left(n-\frac{1-\lambda_{1}}{4}\right)^{2}
$$

We see that for $\lambda_{1} \lambda_{2}=-1$ (i. e. depending on the choice of the splitter), these energy values are not equal to the energies $E_{n}^{\prime}, n=1,2, \cdots$ which determine the peaks of the density of states. Indeed, using Eqs. (40) and (42) we obtain for the relation between density of states and the transmission probability

$$
|t|^{2}=\frac{1}{\lambda_{1} \sigma}\left\{\tilde{t}^{\prime 2}-\frac{\pi \hbar v \varepsilon^{2}}{2 l^{\prime}} \bar{\rho}^{\prime}\right\} .
$$

Using Eqs. (40) and (43) we can show that $\int_{\mathcal{E}_{n}{ }^{2}}^{\mathcal{E}_{n+1}} d E \quad \bar{\rho}^{\prime}=1$. This implies that the condition Eq. (13) is fulfilled also by a wire with a side branch. The different behavior, depending on the sign of $\lambda_{1} \lambda_{2}$, is especially apparent in the week coupling limit. In this limit, for $\lambda_{1} \lambda_{2}=-1$, almost all the particles incident from the wire on the wave splitter pass through the junction without noticing the side branch. Conversely, in the week coupling limit of a wave splitter with $\lambda_{1} \lambda_{2}=1$ almost all the particles incident from the wire on the wave splitter are reflected at the wave splitter. Fig. 11 shows the transmission probability and the density of state as a function of $k l^{\prime}$ for the wire with a side branch connected by a junction with $\lambda_{1}=-1, \lambda_{2}=-1$ and $\varepsilon^{2}=0.35$.

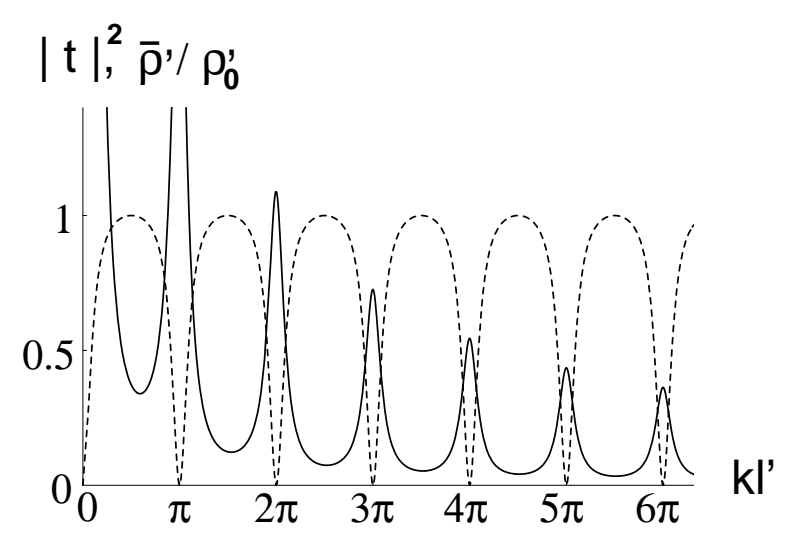

FIG. 11. Transmission probability (the dashed line) and the density of states $\bar{\rho}^{\prime}$ (the solid line) in units of $\rho_{0}^{\prime} \equiv m l^{\prime 2} /\left(2 \pi \hbar^{2}\right)$ for a wire with a side branch.

\section{E. Wire with scattering and a side branch}

The previous two models are examples which demonstrate two different behaviors of the transmission amplitude in the complex plane. These different behaviors are illustrated by Fig. 4 and 5. Clearly, both of these models are very particular (non-generic) and the question arises how the behavior exemplified by these two simple models shows up in more complicated structures. To examine this question we now consider a structure which incorporates both the resonant double barrier and the side branch. A schematic illustration of this system is shown in Fig. 12 .

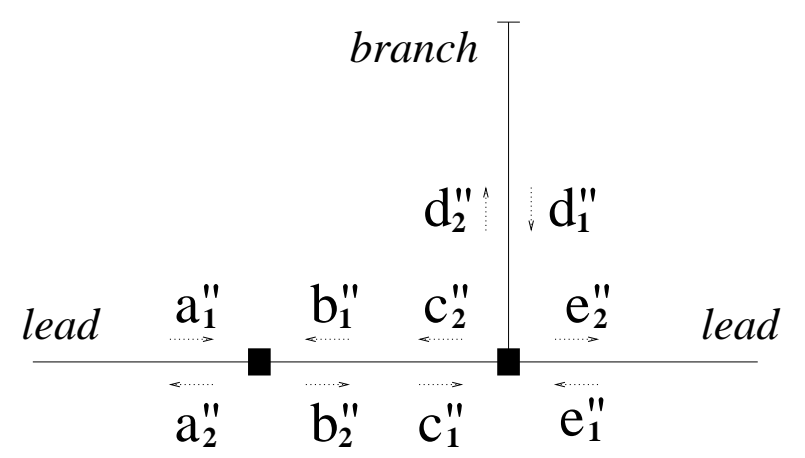

FIG. 12. Current amplitudes for a model with scattering in the wire and with a side branch.

We use the same potential barrier as in the resonant double barrier structure and the same wave splitter as we used for the description of the wire with the side branch. Again we will assume that the potential outside these scatterers is everywhere the same. In this model the current amplitudes $a_{j}^{\prime \prime}, b_{j}^{\prime \prime}, c_{j}^{\prime \prime}, d_{j}^{\prime \prime}$ and $e_{j}^{\prime \prime}, j=1,2$ which are shown in Fig. 12 with the directions, are connected by 
the following relations,

$$
\begin{gathered}
\left(\begin{array}{c}
a_{2}^{\prime \prime} \\
b_{2}^{\prime \prime}
\end{array}\right)=\left(\begin{array}{cc}
\tilde{r} & \tilde{t} \\
\tilde{t} & \tilde{r}
\end{array}\right)\left(\begin{array}{c}
a_{1}^{\prime \prime} \\
b_{1}^{\prime \prime}
\end{array}\right) \\
\left(\begin{array}{c}
b_{1}^{\prime \prime} \\
c_{1}^{\prime \prime}
\end{array}\right)=\left(\begin{array}{cc}
0 & \tau \\
\tau & 0
\end{array}\right)\left(\begin{array}{c}
b_{2}^{\prime \prime} \\
c_{2}^{\prime \prime}
\end{array}\right) \\
\left(\begin{array}{c}
c_{2}^{\prime \prime} \\
d_{2}^{\prime \prime} \\
e_{2}^{\prime \prime}
\end{array}\right)=\left(\begin{array}{ccc}
\tilde{r}^{\prime} & \varepsilon & \tilde{t}^{\prime} \\
\varepsilon & \sigma & \varepsilon \\
\tilde{t}^{\prime} & \varepsilon & \tilde{r}^{\prime}
\end{array}\right)\left(\begin{array}{c}
c_{1}^{\prime \prime} \\
d_{1}^{\prime \prime} \\
e_{1}^{\prime \prime}
\end{array}\right) .
\end{gathered}
$$

Furthermore, as in the model with the side branch we put $d_{1}^{\prime \prime}=\tau^{\prime} d_{2}^{\prime \prime}$.

For the overall scattering matrix of these system we obtain

$$
S=\frac{1}{\tilde{r}^{*} D}\left(\begin{array}{cc}
V & W \\
W & -\lambda_{1} \tau^{\prime} \tau^{2} V^{*}
\end{array}\right)
$$

in which $D, V$ and $W$ are defined by

$$
\begin{gathered}
D \equiv 1-\sigma \tau^{\prime}-\tilde{r} \tilde{r}^{\prime}\left(1+\lambda_{1} \tau^{\prime}\right) \tau^{2} \\
V \equiv D-|\tilde{t}|^{2}\left(1-\sigma \tau^{\prime}\right) \\
W \equiv \tilde{r}^{*} \tilde{t} \tilde{t}^{\prime}\left(1-\lambda_{1} \tau^{\prime}\right) \tau .
\end{gathered}
$$

The transmission amplitude $t$ can be brought into the form

$$
t=\frac{\tilde{t} \tilde{t}^{\prime}\left(1+\lambda_{1} e^{2 i k l^{\prime}}\right) e^{i k l}}{1+\sigma e^{2 i k l^{\prime}}-\tilde{r} \tilde{r}^{\prime}\left(1-\lambda_{1} e^{2 i k l^{\prime}}\right) e^{2 i k l}} .
$$

In this representation the transmission amplitude $t$ depends on the energy $E$ only through the wave vector $k=\sqrt{2 m E} / \hbar$. As a function of energy, the path of this transmission amplitude in the complex plane is shown in Fig. 3. Here, the parameters chosen for Fig. 3 are $\tilde{\theta}=2.2, \tilde{\phi}=2.1, \lambda_{1}=-1, \lambda_{2}=1, \varepsilon^{2}=0.35, l=1$ and $l^{\prime}=4$. This figure shows clearly that a more general model combines the behavior of the resonant double barrier model and the stub model. Sequences of turns of the transmission amplitude which path through the origin are interrupted by "double turns" characteristic of the resonant double barrier in which the transmission amplitude is non-zero. From Eq. (52) it can be noted that the wire with scattering and with a side branch has the same zero points for the transmission amplitude as the wire with the side branch.

\section{CONCLUSIONS}

In this work we have discussed the transmission amplitude as function of energy in the complex plane for scattering systems (without a magnetic field) connected to two single channel leads. We emphasize that there are two phases which are of importance, namely the phase which appears in the Friedel sum rule and the phase of the transmission amplitudes. Except in special cases, these two phases are in general different This important point has also been emphasized by Lee.27 The two phases are different if the transmission amplitude exhibits an energy at which it is zero. At these energies the transmission amplitude phase and the Friedel phase acquire an additional difference given by $\pm \pi$. If the transmission amplitude exhibits no zero point between resonances the variation of the phase from one resonant peak to another is close to $\pi$. If a zero point exists the phase change between consecutive resonance peaks of the transmission probability is close to $2 \pi$. This difference is shown clearly in the paths of the transmission amplitude in the complex plane.

For a sufficiently general model, we expect sequences of resonant peaks which are in phase (the phase increases by $2 \pi$ ) interrupted by peaks which are out of phase (the phase increases only by $\pi$ as we go from one peak to the next). In terms of the parity of the Aharonov-Bohm effect, these implies sequences of peaks over which the parity is conserved. These sequences are interrupted by transitions which generate a flip in the parity. It is clearly desirable to investigate now a number of statistical questions: For example for a fully chaotic quantum dot one would like to find the ensemble averaged density of zero's and compare this with the ensemble averaged density of states. Furthermore, one would like to know if such a cavity exhibits correlations in the occurrence of zero's (long sequences of zero's interrupted by a flip in the parity), etc. Since the distribution of eigenvalues is known, random matrix theory likely gives an answer to these questions.

We add a remark on Fano resonances: Fano resonances arise due to the coupling of discrete states with continuous states. Such resonances also exhibit transmission zero's 4042 Consequently, for such resonances the Friedel phase also does not coincide with the phase changes of the transmission amplitudes. The wire with a side branch investigated here also couples a set of discrete states with a continuum and thus provides for interfering transmission paths. However, the resonances in this case are not of the Fano type (as shown in Fig. 11) but rather Breit-Wigner resonances in the reflection probability.

It is very interesting to investigate the behavior of the transmission amplitude in the complex plane for variations in parameters other than the energy. For instance we can ask about the path of the transmission amplitude if the AB flux increases by a flux quantum in a multiply connected geometry. The additional questions raised in this section clearly demonstrate that the investigation of the path of the transmission amplitude in the complex plane is an interesting avenue of future research. 


\section{ACKNOWLEDGMENTS}

We are grateful to A. Levy Yeyati, M. Devoret and D. Esteve for stimulating discussions. T.T acknowledges the support of the Swiss Federal government. M. B. is supported by the Swiss National Science foundation.

\section{APPENDIX A: RELATION BETWEEN PHASES FOR THE WIRE WITH A SIDE BRANCH}

In this Appendix we outline the derivation of Eq. (38) which relates the Friedel phase and transmission amplitude phase for the wire with a side branch. First, using Eqs. (33) and (36) we find

$$
\bar{t}_{ \pm}=\frac{-\lambda_{1}-\sigma}{2} \frac{1-\lambda_{1} e^{i \varphi^{\prime}}}{1-\sigma e^{i \varphi^{\prime}}} \pm \eta
$$

The transmission amplitude $t$ is dependent on the energy only through the quantity $\varphi^{\prime}$, so that we obtain

$$
\frac{\partial \theta^{(t)}}{\partial E}=\lim _{\eta \rightarrow+0} \frac{\partial \varphi^{\prime}}{\partial E} \frac{\partial \operatorname{Arg}\left\{\bar{t}_{ \pm}\right\}}{\partial \varphi^{\prime}} .
$$

Using Eq. (A1) the derivative $\partial \operatorname{Arg}\left\{\bar{t}_{ \pm}\right\} / \partial \varphi^{\prime}$ is found to be

$$
\begin{aligned}
& \frac{\partial \operatorname{Arg}\left\{\bar{t}_{ \pm}\right\}}{\partial \varphi^{\prime}} \\
= & \left\{1+\left(\frac{\operatorname{Im}\left\{\bar{t}_{ \pm}\right\}}{\operatorname{Re}\left\{\bar{t}_{ \pm}\right\}}\right)^{2}\right\}^{-1} \frac{\partial}{\partial \varphi^{\prime}} \frac{\operatorname{Im}\left\{\bar{t}_{ \pm}\right\}}{\operatorname{Re}\left\{\bar{t}_{ \pm}\right\}} \\
= & \frac{1}{2} \frac{1-\sigma^{2}}{1+\sigma^{2}-2 \sigma \cos \varphi^{\prime}} \\
& \times \frac{\mp 2 f_{1}\left(\sigma, \varphi^{\prime}\right) \eta+f_{3}\left(\sigma, \lambda_{1}, \varphi^{\prime}\right)}{2 f_{2}\left(\sigma, \varphi^{\prime}\right) \eta^{2}+\left(1 \mp 2 \lambda_{1} \eta\right) f_{3}\left(\sigma, \lambda_{1}, \varphi^{\prime}\right)} \\
= & \frac{1}{2} \frac{1-\sigma^{2}}{1+\sigma^{2}-2 \sigma \cos \varphi^{\prime}} \\
& +\frac{1}{2} F\left(1 \mp 2 \lambda_{1} \eta, \lambda_{1} \sigma, \varphi^{\prime}+\frac{1+\lambda_{1}}{2} \pi\right)
\end{aligned}
$$

where the functions $f_{1}(x, y), f_{2}(x, y)$ and $f_{3}(x, y, z)$ are defined by

$$
\begin{gathered}
f_{1}(x, y) \equiv 2 x-\left(1+x^{2}\right) \cos y \\
f_{2}(x, y) \equiv 1+x^{2}-2 x \cos y \\
f_{3}(x, y, z) \equiv(1+x y)^{2}(1-y \cos z)
\end{gathered}
$$

and the function $F(x, y, z)$ is defined by

$$
\begin{aligned}
& F(x, y, z) \\
& \equiv \frac{\left(1-x^{2}\right)\left(1-y^{2}\right)}{(1-x)^{2}(1-y)^{2}+2(1+x y)(x+y)(1+\cos z)}
\end{aligned}
$$

$F(x, y, z)$ has the following properties. First it follows that

$$
\begin{aligned}
& \lim _{x \rightarrow 1 \pm 0} F(x, y, z) \\
& =\left\{\begin{array}{cc}
0 & \text { in } z=(2 n+1) \pi \\
\mp \epsilon\left(1-y^{2}\right) \times \infty & \text { in } z \neq(2 n+1) \pi,
\end{array}\right.
\end{aligned}
$$

$n=0, \pm 1, \pm 2, \cdots$. Second it follows that

$$
\begin{aligned}
& \int_{\lambda}^{\lambda+2 \pi} d z F(x, y, z) \\
& =\frac{1}{i} \oint_{C} d \omega \frac{\left(1-x^{2}\right)\left(1-y^{2}\right)}{\{(1+x y) \omega+x+y\}\{(x+y) \omega+1+x y\}} \\
& =\left\{\begin{array}{l}
+2 \pi \text { in } 0 \neq|x+y|<|1+x y| \\
-2 \pi \text { in }|x+y|>|1+x y| \neq 0
\end{array}\right.
\end{aligned}
$$

where $\lambda$ is a real number and $\omega \equiv \exp (i z)$. $C$ is the path running counterclockwise on the circle whose center is the origin and the radius is 1. Eqs. (A8) and A9 lead to

$$
\begin{aligned}
& \lim _{x \rightarrow 1 \pm 0} F(x, y, z) \\
& =\mp 2 \pi \epsilon\left(1-y^{2}\right) \sum_{n=-\infty}^{+\infty} \delta(z-(2 n+1) \pi) .
\end{aligned}
$$

Using Eq. (34) and the inequality $E>0$, we obtain

$$
\delta\left(\varphi^{\prime}+\frac{1+\lambda_{1}}{2} \pi-(2 n+1) \pi\right)=\left(\frac{\partial \varphi^{\prime}}{\partial E}\right)^{-1} \delta\left(E-E^{(n)}\right) .
$$

From Eqs. A2 , A3), (A10) and (A11) we derive

$$
\frac{\partial \theta^{(t)}}{\partial E}=\frac{\partial \varphi^{\prime}}{\partial E}\left|\frac{\varepsilon}{1-\sigma \tau^{\prime}}\right|^{2} \pm \lambda_{1} \pi \sum_{n=1}^{+\infty} \delta\left(E-E^{(n)}\right) .
$$

Using Eqs. (35) and (A12) we obtain Eq. (38).

${ }^{1}$ R. G. Newton, Scattering Theory of Wave and Particles (Springer Verlag, Town, year missing).

2 J. Friedel, Phil. Mag. 43, 153 (1952).

3 J. S. Langer and V. Ambegaokar, Rev. 121, 1090 (1961).

${ }^{4}$ D. C. Langreth, Phys. Rev. 150, 517 (1966).

${ }^{5}$ R. Dashen, S. H. Ma and H. J. Bernstein, Phys. Rev. 187, 345 (1969).

${ }^{6}$ F. Kassubek, C. A. Stafford and H. Grabert, Phys. Rev. B 59, 7560 (1999).

${ }^{7}$ E. Akkermans, A. Auerbach, J. E. Avron, and B. Shapiro, Phys. Rev. Lett. 66, 76 (1991).

8 T. Christen, Phys. Rev. B55, 706 (1997); V. Gasparian, T. Christen and M. Büttiker, Phys. Rev. A 54, 4022 (1996). 
${ }^{9}$ V. A. Gopar, P. A. Mello, and M. Büttiker, Phys. Rev. Lett. 77, 3005 (1996).

${ }^{10} \mathrm{M}$. Buttiker and A. M. Martin (unpublished) condmat/9902320.

${ }^{11}$ A. Yacoby, M. Heiblum, D. Mahalu and H. Shtrikman, Phys. Rev. Lett. 74, 4047 (1995).

12 R. Schuster, E. Buks, M. Heiblum, D. Mahalu, V. Umansky and H. Shtrikman, Nature, 385, 417 (1997).

${ }^{13}$ A. Levy Yeyati and M. Büttiker, Phys. Rev. B 52, R14360 (1995).

${ }^{14}$ G. Hackenbroich and H. A. Weidenmüller, Phys. Rev. Lett. 76, 110 (1996).

${ }^{15}$ C. Bruder, R. Fazio and H. Schoeller, Phys. Rev. Lett. 76, 114 (1996).

${ }^{16}$ A. Yacoby, R. Schuster and M. Heiblum, Phys. Rev. B 53, 9583 (1996).

${ }^{17}$ G. Hackenbroich and H. A. Weidenmüller, Phys. Rev. B 53, 16379 (1996).

${ }^{18}$ P. S. Deo and A. M. Jayannavar, Mod. Phys. Lett, B 10, 787 (1996).

${ }^{19}$ Y. Oreg and Y. Gefen, Phys. Rev. B 55, 13726 (1997).

${ }^{20}$ G. Hackenbroich and H. A. Weidenmüller, Europhys. Lett, 38 (2), 129 (1997).

${ }^{21}$ G. Hackenbroich, W. D. Heiss and H. A. Weidenmüller, Phys. Rev. Lett. 79, 127 (1997).

${ }^{22}$ H. Xu and W. Sheng, Phys. Rev. B 57, 11903 (1998).

${ }^{23}$ C. - Mo Ryu and S. Y. Cho, Phys. Rev. B 58, 3572 (1998).

${ }^{24}$ R. Baltin, Y. Gefen, G. Hackenbroich and H. A. Weidenmüller, cond-mat/9807286.

${ }^{25}$ K. Kang, cond-mat/9811354.

${ }^{26}$ H. Q. Xu (unpublish).
${ }^{27}$ H. -W. Lee, Phys. Rev. Lett. 82, 2223 (1999).

${ }^{28}$ P. G. Silvestrov and Y. Imry, cond-mat/9903299.

${ }^{29}$ A. Levy Yeyati, informal July, 1996 correspondence to M. B. Ley Yeyati investigated the transmission amplitude of dots described by lattice models.

${ }^{30}$ C. J. B. Ford, A. B. Fowler, J. M. Hong, C. M. Knoedler, S. E. Laux, J. J. Weiner and S. Washburn, Surface Science 229, 307 (1990).

${ }^{31}$ M. Büttiker, IBM J. Res. Developm. 32, 63 (1988).

${ }^{32}$ H.-L. Engquist and P. W. Anderson, Phys. Rev. B 24, 1151 (1981).

33 M. Büttiker, Y. Imry and M. Ya. Azbel, Phys. Rev. A 30 1982 (1984).

${ }^{34}$ F. Sols, M. Macucci, U. Ravaioli and K. Hess, Appl. Phys. Lett. 54, 350 (1989).

35 S. Subramanian, S. Bandyopadhyay and W. Porod, J. Appl. Phys. 68, 4861 (1990).

${ }^{36}$ M. Büttiker, Physica Scripta, T54, 104 (1994).

${ }^{37}$ P. Cedraschi and M. Büttiker, J. Phys. Cond-matt. 10, 3985 (1998).

${ }^{38}$ Z. -an Shao, W. Porod, and C. S. Lent, Phys. Rev. B 49, 7453 (1994).

${ }^{39}$ P. S. Deo and A. M. Jayannavar, Phys. Rev. B 50 (1994) 11619.

${ }^{40}$ U. Fano, Phys. Rev. 124, B1866 (1961).

${ }^{41}$ As one of recent papers about Fano resonances in quantum transport phenomena see the following paper and the references therein: J. U. Nöckel and A. D. Stone, Phys. Rev. B 50, 17415 (1994).

42 J. Goeres et al. report the observation of Fano-Resoances in almost open quantum dots (unpublished). 